\title{
Neurally Adjusted Ventilatory Assist After Pediatric Cardiac Surgery: Clinical Experience and Impact on Ventilation Pressures
}

\author{
Benjamin Crulli MD, Mariam Khebir, Baruch Toledano MD MSc, Suzanne Vobecky MD, \\ Nancy Poirier MD, and Guillaume Emeriaud MD PhD
}

\begin{abstract}
BACKGROUND: After pediatric cardiac surgery, ventilation with high airway pressures can be detrimental to right ventricular function and pulmonary blood flow. Neurally adjusted ventilatory assist (NAVA) improves patient-ventilator interactions, helping maintain spontaneous ventilation. This study reports our experience with the use of NAVA in children after a cardiac surgery. We hypothesize that using NAVA in this population is feasible and allows for lower ventilation pressures. METHODS: We retrospectively studied all children ventilated with NAVA (invasively or noninvasively) after undergoing cardiac surgery between January 2013 and May 2015 in our pediatric intensive care unit. The number and duration of NAVA episodes were described. For the first period of invasive NAVA in each subject, detailed clinical and ventilator data in the $\mathbf{4} \mathbf{h}$ before and after the start of NAVA were extracted. RESULTS: 33 postoperative courses were included in 28 subjects with a median age of 3 [interquartile range (IQR) 1-12] months. NAVA was used invasively in 27 courses for a total duration of 87 (IQR 15-334) h per course. Peak inspiratory pressures and mean airway pressures decreased significantly after the start of NAVA (mean differences of $5.8 \mathrm{~cm} \mathrm{H}_{2} \mathrm{O}(95 \% \mathrm{CI} 4.1-7.5)$ and $2.0 \mathrm{~cm} \mathrm{H} \mathrm{H}_{2} \mathrm{O}(95 \%$ CI 1.2-2.8), respectively, $P<.001$ for both). There was no significant difference in vital signs or blood gas values. NAVA was used noninvasively in 14 subjects, over 79 (IQR 25-137) h. CONCLUSIONS: NAVA could be used in pediatric subjects after cardiac surgery. The significant decrease in airway pressures observed after transition to NAVA could have a beneficial impact in this specific population, which should be investigated in future interventional studies. Key words: respiratory support; noninvasive ventilation; mechanical ventilation; patient-ventilator interaction; cardio-respiratory interaction; patient-ventilator asynchrony; pediatric; cardiac surgery. [Respir Care 2018;63(2):208-214. (C) 2018 Daedalus Enterprises]
\end{abstract}

\section{Introduction}

Assisted ventilation is essential to the care of children after cardiac surgery, sometimes for prolonged periods of

\footnotetext{
Drs Crulli, Toledano, and Emeriaud, as well as Ms Khebir, are affiliated with the Pediatric Intensive Care Unit, CHU Sainte-Justine, Université de Montréal, Montréal, Québec, Canada. Drs Vobecky and Poirier are affiliated with the Department of Pediatric Cardiovascular Surgery, CHU Sainte-Justine, Université de Montréal, Montréal, Quebec, Canada.
}

Parts of the results of this study were presented by Benjamin Crulli at the 2016 Reanimation Congress, held January 13-15, 2016, in Paris, France.

Dr Emeriaud holds a Clinical Research Scholarship from the Fonds de la Recherche en Santé du Québec. He is also the principal investigator of an ongoing clinical study which is financially supported by Maquet Critical Care. He does not receive any salary nor any personal financial support time. Although positive-pressure ventilation can reduce the work of breathing and decrease afterload of the left ventricle, intrathoracic pressures can also reduce venous return and increase the afterload of the right ventricle. ${ }^{1-3}$ In the management of patients at risk for right ventricle failure or with a passive pulmonary blood flow, minimizing the ventilatory pressure is therefore crucial. In addition, support that is excessive or asynchronous can exacerbate

\footnotetext{
from this company. The other authors have disclosed no conflicts of interest.

Correspondence: Guillaume Emeriaud MD PhD, CHU Sainte-Justine, Université de Montréal, 3175 Chemin de la Côte-Sainte-Catherine, Montréal, QC H3T 1C5, Canada. E-mail: guillaume.emeriaud@umontreal.ca.
}

DOI: $10.4187 /$ respcare. 05625 
the known complications of mechanical ventilation. ${ }^{4-6} \mathrm{Neu}-$ rally adjusted ventilatory assist (NAVA) is a ventilation mode that provides ventilatory support that is synchronous and proportional to the electrical activity of the diaphragm $\left(\mathrm{EA}_{\mathrm{di}}\right)$, allowing variation in tidal volume and inspiratory time between breaths. ${ }^{7}$ Many studies have shown that NAVA improves patient-ventilator interactions in children thereby permitting more spontaneous ventilation. ${ }^{8-14}$ The use of NAVA after pediatric cardiac surgery has been rarely reported in the literature, ${ }^{15,16}$ but it is regularly used in our pediatric intensive care unit (PICU) because of its theoretical advantages in this specific population.

In this article, we describe and analyze this experience. The main objective is to assess the feasibility of invasive NAVA in children admitted to PICU after heart surgery and its impact on ventilation pressures. The secondary objectives are to evaluate its effect on clinical and paraclinical parameters and to describe the use of noninvasive NAVA after extubation in this population. We hypothesize that using NAVA in this population is feasible and allows for lower ventilation pressures.

\section{Methods}

\section{Study Design}

This retrospective study was conducted in the PICU of CHU Sainte-Justine Hospital, in Montreal, Quebec, from January 8, 2013, to May 31, 2015. Local ethics board approval was obtained with a waiver of consent (\#4175).

\section{Population}

All subjects under $18 \mathrm{y}$ of age who were admitted to the PICU following heart surgery and who were ventilated using NAVA (invasively or not) at least once during their PICU stay were included. Eligible patients were identified by automatically extracting all NAVA settings from the PICU electronic medical record system. When a subject underwent multiple cardiac surgeries during the same hospitalization, each postoperative course was considered independently. For each postoperative course, the duration of the different ventilation periods in each mode was noted.

During the study period, a limited number of ventilators in the PICU had NAVA capability (2 machines until June 2013, then 4 machines). Use of NAVA and the choice of settings were at the discretion of the medical team, depending on ventilator availability. Although no written protocol for the choice of ventilation mode was used in the PICU during this period, a written protocol was available to guide the management of NAVA. This protocol suggested to first estimate the level of $\mathrm{EA}_{\mathrm{di}}$ that would be expected under NAVA, based on the $\mathrm{EA}_{\mathrm{di}}$ observed before the switch, the change that was expected or desired (eg, if

\section{QUICK LOOK}

\section{Current knowledge}

High positive airway pressure can be detrimental after pediatric cardiac surgery. Neurally adjusted ventilatory assist (NAVA) improves patient-ventilator interactions and facilitates the maintenance of the patient's own ventilation.

\section{What this paper contributes to our knowledge}

NAVA was feasible and well tolerated in our experience after pediatric cardiac surgery. The transition to NAVA was followed by a marked decrease in ventilation pressures, while adequate ventilation support was maintained.

the patient seemed to be over-assisted with low $\mathrm{EA}_{\mathrm{di}}$, an increase in $\mathrm{EA}_{\mathrm{di}}$ would be expected), and the consideration that normal $\mathrm{EA}_{\mathrm{di}}$ in critically ill children is usually between 5 and $20 \mu \mathrm{V} .{ }^{17}$ Subsequently, the initial NAVA level was calculated as the ratio between expected driving pressure (usually the one observed in conventional ventilation) divided by the expected $\mathrm{EA}_{\mathrm{di}}$. Most importantly, the NAVA level was reassessed after the transition to NAVA and adjusted based on the observed $\mathrm{EA}_{\mathrm{di}}$, the subject's respiratory efforts and breathing frequency, the tidal volume (usual target, $5-8 \mathrm{~mL} / \mathrm{kg}$ ), and minute ventilation. The weaning procedure was at the discretion of the treating team and was not protocolized.

\section{Data Collection}

Data were collected retrospectively through a review of the electronic charts. Baseline data points included demographics, history of chronic pulmonary disease, duration of cardiopulmonary bypass, duration of aortic crossclamping, and length of PICU stay. The type of cardiac surgery was recorded, in particular regarding the risk of right ventricular failure or low pulmonary flow (eg, Glenn, Fontan, Blalock-Taussig shunt, or other surgery), which was assessed by 2 investigators (GE and BT). The number, timing, and duration of all NAVA episodes were documented. The first period of invasive NAVA in each postoperative course was looked at in more detail; during the 4-h periods preceding and following the transition to NAVA, ventilation settings and measured values [peak inspiratory pressure (PIP), mean airway pressure $\left(\overline{\mathrm{P}}_{\mathrm{aw}}\right)$, tidal volume], vital signs (average breathing frequency, average heart rate, average mean arterial blood pressure, $\mathrm{S}_{\mathrm{pO}_{2}}$ ), and available arterial or capillary blood gas data $\left(\mathrm{pH}, \mathrm{P}_{\mathrm{aCO}_{2}}, \mathrm{P}_{\mathrm{aO}}\right.$, lactates) were recorded. 


\section{Outcome Measures}

The primary outcome measure was the hourly evolution of PIP and $\overline{\mathrm{P}}_{\mathrm{aw}}$ surrounding the start of NAVA. Secondarily, the change in clinical status after the transition to NAVA (pre-post comparison) was studied. As an indirect correlate for the feasibility of NAVA in clinical practice, NAVA episodes that were rapidly terminated (after $<2 \mathrm{~h}$ ) were accounted for.

\section{Statistical Analysis}

Statistical analysis was performed using SPSS Statistics 23 (IBM). Categorical variables are reported with absolute numbers and percentages. For continuous variables, we tested the normality of their distribution using the ShapiroWilk test. Continuous variables are reported using means $\pm \mathrm{SD}$ or medians with interquartile range (IQR, ie, 25th-75th percentile), depending on the distribution. With regard to the primary outcome (hourly PIP and $\overline{\mathrm{P}}_{\mathrm{aw}}$ ), a one-way repeated measures analysis of variance test was performed to determine whether there was a significant difference between the 8 time points, with post hoc complex contrasts allowing for comparison between the different time points. A $P$ value $<.05$ was considered significant. Other secondary clinical values were compared with paired sample $t$ tests when the variables were normally distributed, and with Wilcoxon signed rank tests otherwise.

\section{Results}

During the study period, 557 patients underwent cardiac surgery in our center. Twenty-eight subjects were ventilated using NAVA over 33 postoperative courses (Fig. 1) and were included in the analysis. Of these, NAVA was used during invasive ventilation only in 19 courses, during noninvasive ventilation only in 6 courses, and during both invasive and noninvasive ventilation in 8 courses.

\section{Characteristics of NAVA Episodes}

As described in Table 1, subjects had a median age of 3 (IQR 1-12) months and stayed in intensive care for a median duration of 38 (IQR 23-76) d. Forty-three percent of the courses followed surgery considered at high risk for right-sided heart failure or low pulmonary flow. Various characteristics of the NAVA episodes are described in Table 2. The subjects underwent a median number of 2 (IQR 1-2) episodes of invasive NAVA within their postoperative course, with the first episode starting 8.3 (IQR 2.6-13.7) d after surgery. Before switching to NAVA, 17 subjects $(63 \%)$ were ventilated using pressure-control ventilation, 8 subjects $(30 \%)$ using volume-control ventilation,

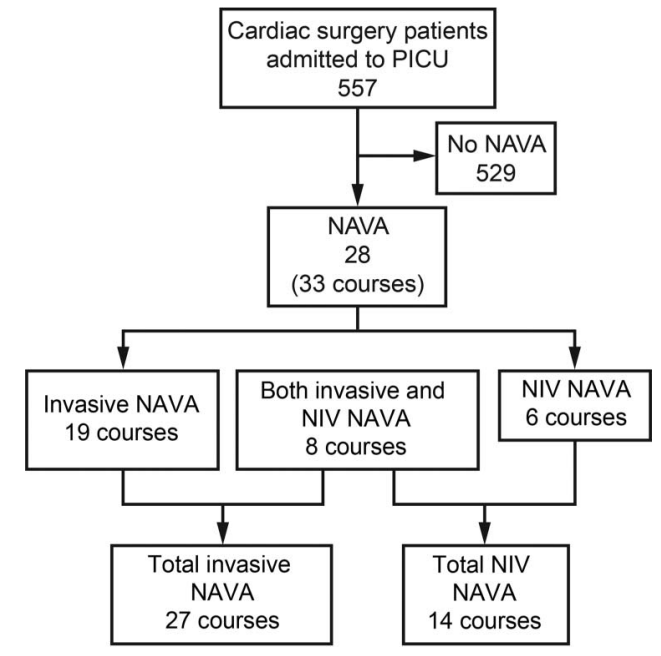

Fig. 1. Flow chart. PICU = pediatric intensive care unit, NAVA = neurally adjusted ventilatory assist, NIV = noninvasive ventilation

Table 1. Subject Characteristics

\begin{tabular}{|c|c|}
\hline Age, median (IQR) months & $3(1-12)$ \\
\hline Weight, median (IQR) kg & $5.6(3.5-8.3)$ \\
\hline Male, $n(\%)$ & $16(48 \%)$ \\
\hline History of chronic pulmonary disease, $n(\%)$ & $3(9 \%)$ \\
\hline \multicolumn{2}{|l|}{ Type of cardiac surgery, $n(\%)$} \\
\hline Glenn & $2(6 \%)$ \\
\hline Fontan & $1(3 \%)$ \\
\hline Blalock-Taussig shunt & $2(6 \%)$ \\
\hline Other surgery at risk for right-sided heart failure & $9(27 \%)$ \\
\hline $\begin{array}{l}\text { Surgery not at particular risk for right-sided } \\
\text { heart failure or low pulmonary blood flow }\end{array}$ & $19(57 \%)$ \\
\hline Duration of cardiopulmonary bypass, median (IQR) min* & $130(107-174$ \\
\hline Duration of aortic cross-clamping, mean $\pm \mathrm{SD} \min \dagger$ & $87 \pm 49$ \\
\hline PICU length of stay, median (IQR) d & $38(23-76)$ \\
\hline \multicolumn{2}{|c|}{$\begin{array}{l}\text { Thirty-three postoperative courses. Continuous data are reported as mean } \pm \mathrm{SD} \text { for normally } \\
\text { distributed variables and as median (IQR) for non-normally distributed variables. } \\
\begin{array}{ll}* n=15 \\
\dagger n=14 \\
\text { IQR }=\text { interquartile range } \\
\text { PICU = pediatric intensive care unit }\end{array}\end{array}$} \\
\hline
\end{tabular}

and 2 subjects (7\%) using pressure support ventilation. The NAVA level, initially set at $1.4 \pm 0.6 \mathrm{~cm} \mathrm{H}_{2} \mathrm{O} / \mu \mathrm{V}$, was at $1.3 \pm 0.6 \mathrm{~cm} \mathrm{H}_{2} \mathrm{O} / \mu \mathrm{V}$ after $4 \mathrm{~h}$. One subject was treated with NAVA for $<2 \mathrm{~h}$ in total.

Noninvasive NAVA was used, on average, $12.8 \pm 10.0 \mathrm{~d}$ after surgery, for a duration equivalent to $53 \%$ (IQR $30-100 \%$ ) of the total noninvasive ventilation period. Noninvasive NAVA resulted in successful weaning of ventilation (ie, no need for re-intubation) in $71 \%$ of cases.

\section{Primary Outcome}

The evolution of ventilation pressures (PIP and $\overline{\mathrm{P}}_{\mathrm{aw}}$ ) after the start of NAVA is depicted in Figure 2. A significant 
Table 2. Characteristics of NAVA Episodes

\begin{tabular}{|c|c|}
\hline \multicolumn{2}{|l|}{ Invasive NAVA (27 courses) } \\
\hline $\begin{array}{l}\text { Time between surgery and NAVA initiation, } \\
\text { median (IQR) d }\end{array}$ & $8.3(2.6-13.7)$ \\
\hline Number of episodes per course, median (IQR) & $2(1-2)$ \\
\hline Time spent in invasive NAVA, median (IQR) d & $3.6(0.6-13.9)$ \\
\hline Number of episodes lasting $<2 \mathrm{~h}, n(\%)$ & $7(14 \%)$ \\
\hline $\begin{array}{l}\text { Number of courses with total NAVA duration } \\
\quad<2 \mathrm{~h}, n(\%)\end{array}$ & $1(4 \%)$ \\
\hline \multicolumn{2}{|l|}{ Noninvasive NAVA (14 courses) } \\
\hline $\begin{array}{l}\text { Time between surgery and noninvasive NAVA, } \\
\text { mean } \pm \text { SD d }\end{array}$ & $12.8 \pm 10.0$ \\
\hline Time spent in noninvasive NAVA, median (IQR) d & $3.3(1.0-5.7)$ \\
\hline $\begin{array}{l}\text { Proportion of total NIV time spent with NAVA, } \\
\text { median (IQR) }\end{array}$ & $53 \%(30-100 \%)$ \\
\hline \multicolumn{2}{|l|}{ NIV outcome } \\
\hline Successful weaning, $n(\%)$ & $10(71 \%)$ \\
\hline NIV failure and re-intubation, $n(\%)$ & $4(29 \%)$ \\
\hline \multicolumn{2}{|l|}{$\begin{array}{l}\text { NAVA }=\text { neurally adjusted ventilatory assist } \\
\text { IQR }=\text { interquartile range } \\
\text { NIV = noninvasive ventilation }\end{array}$} \\
\hline
\end{tabular}

change of PIP and $\overline{\mathrm{P}}_{\mathrm{aw}}$ over time was observed $(P<.001$ for both, one-way repeated measures ANOVA). No change of pressure was observed among the 4-h periods in each mode (all $P>.99$, during both conventional ventilation and NAVA). Conversely, the 4-h average of PIP pre-NAVA $\left(20.9 \pm 5.2 \mathrm{~cm} \mathrm{H}_{2} \mathrm{O}\right.$ ) was significantly higher than the average observed during the 4-h NAVA period (15.1 $\pm 4.9 \mathrm{~cm} \mathrm{H}_{2} \mathrm{O}$ ), with a mean difference of $5.8 \mathrm{~cm}$ $\mathrm{H}_{2} \mathrm{O}$ (95\% CI 4.1-7.5; $\left.P<.001\right)$. A similar difference was observed for $\overline{\mathrm{P}}_{\mathrm{aw}}$, from $11.3 \pm 2.9 \mathrm{~cm} \mathrm{H}_{2} \mathrm{O}$ during the pre-NAVA period to $9.3 \pm 2.8 \mathrm{~cm} \mathrm{H}_{2} \mathrm{O}$ during NAVA, with a mean difference of $2.0 \mathrm{~cm} \mathrm{H}_{2} \mathrm{O}$ (95\% CI 1.2-2.8; $P<.001)$.

As illustrated in Figure 3, the pattern of ventilation pressure change was similar whether the subjects were at risk of right ventricular dysfunction or low pulmonary flow, and whether NAVA was used in the first $3 \mathrm{~d}$ after surgery or later in the PICU course.

\section{Secondary Outcomes}

As shown in Table 3, vital signs and blood lactate concentration did not change significantly after the switch to NAVA in subjects ventilated invasively. Breathing frequencies, tidal volumes, blood $\mathrm{pH}$, and $\mathrm{P}_{\mathrm{aCO}_{2}}$ were not significantly different during the $4 \mathrm{~h}$ preceding NAVA than in the $4 \mathrm{~h}$ after the change in mode. Oxygenation was also stable, as indicated by the similarity of the $\mathrm{S}_{\mathrm{pO}_{2}} / \mathrm{F}_{\mathrm{IO}_{2}}$ and $\mathrm{P}_{\mathrm{aO}_{2}} / \mathrm{F}_{\mathrm{IO}_{2}}$ ratios (in sub-

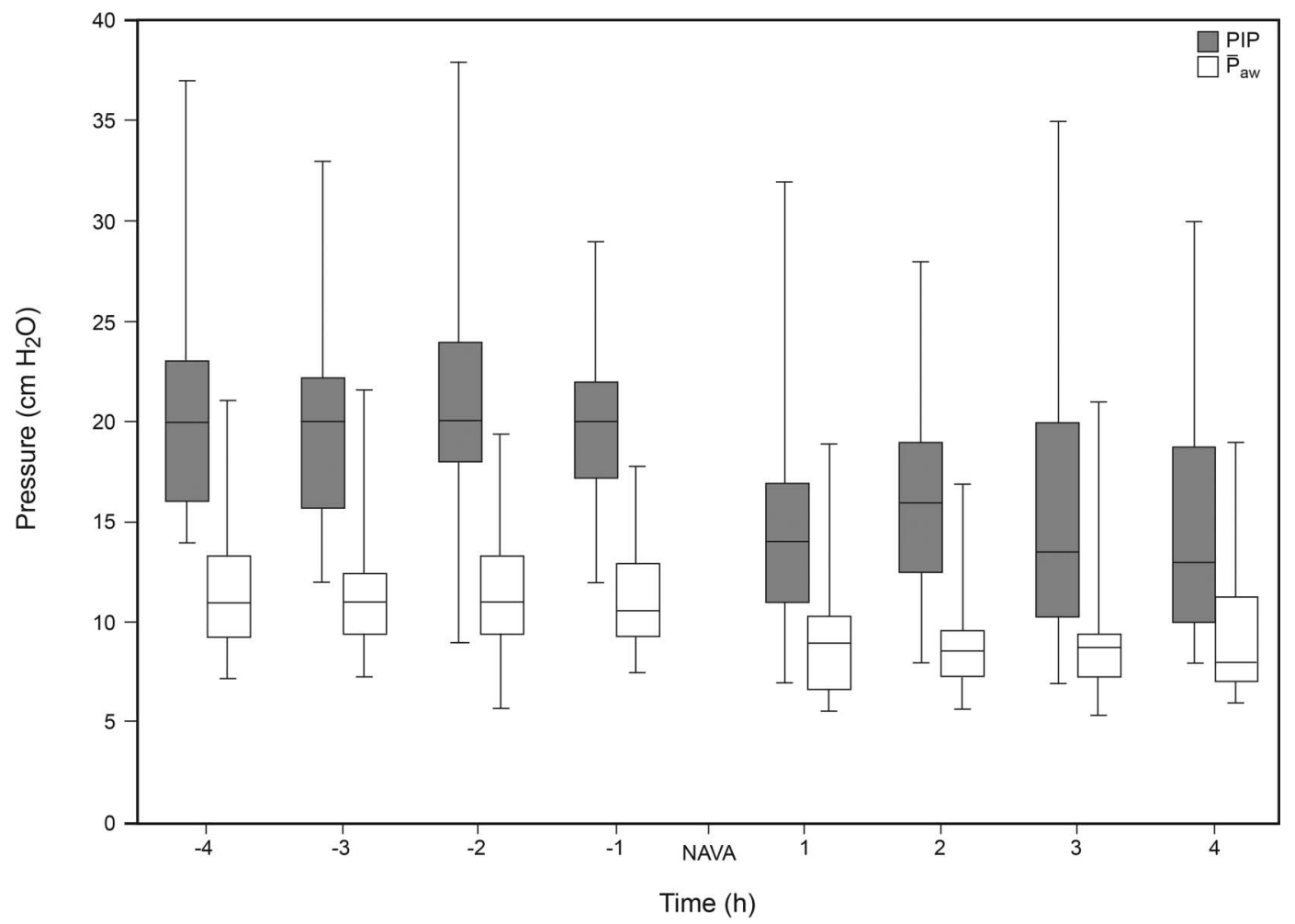

Fig. 2. Evolution of peak inspiratory pressure (PIP) and mean airway pressure $\left(\bar{P}_{a w}\right)$ in the 4 h preceding and following the start of neurally adjusted ventilatory assist (NAVA). Box plots illustrate the median values, interquartile range, and spread. 

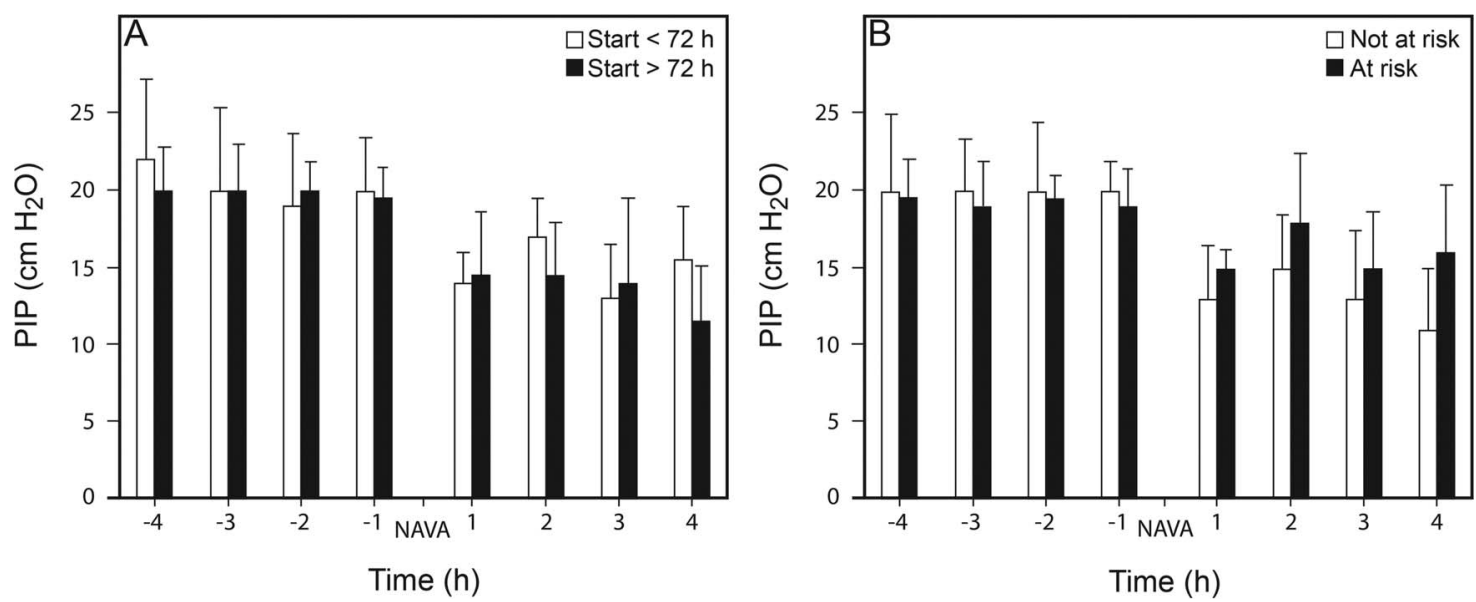

Fig. 3. Evolution of peak inspiratory pressure (PIP) in the $4 \mathrm{~h}$ preceding and following the start of neurally adjusted ventilatory assist (NAVA) depending on the timing of NAVA initiation (before or after $72 \mathrm{~h}$ postadmission, $\mathrm{A}$ ) or the presence of risk of right ventricular failure or low pulmonary flow (B). Data are presented as median with interquartile ranges.

Table 3. Vital Signs and Laboratory Values Before and After Invasive NAVA Initiation

\begin{tabular}{|c|c|c|c|c|c|}
\hline & $n$ & 4-h Period Before NAVA & $n$ & 4-h Period With NAVA & $P$ \\
\hline \multicolumn{6}{|l|}{ Vital signs } \\
\hline Frequency, mean $\pm \mathrm{SD}$ breaths/min & 27 & $31 \pm 8$ & 27 & $31 \pm 9$ & .81 \\
\hline Heart rate, mean $\pm \mathrm{SD}$ beats/min & 27 & $128 \pm 24$ & 27 & $128 \pm 23$ & .98 \\
\hline Mean arterial blood pressure, mean $\pm \mathrm{SD} \mathrm{mm} \mathrm{Hg}$ & 19 & $63 \pm 10$ & 19 & $61 \pm 9$ & .17 \\
\hline \multicolumn{6}{|l|}{ Oxygenation } \\
\hline $\mathrm{F}_{\mathrm{IO}_{2}}$, mean $\pm \mathrm{SD} \%$ & 27 & $52 \pm 22$ & 27 & $52 \pm 23$ & .75 \\
\hline $\mathrm{S}_{\mathrm{pO}_{2}}$, mean $\pm \mathrm{SD} \%$ & 27 & $96 \pm 7$ & 27 & $96 \pm 7$ & .49 \\
\hline $\mathrm{S}_{\mathrm{pO}_{2}} / \mathrm{F}_{\mathrm{IO}_{2}}$ ratio, median (IQR) & 27 & $194(157-275)$ & 27 & $198(165-287)$ & .09 \\
\hline \multicolumn{6}{|l|}{ Ventilation } \\
\hline Tidal volume, mean $\pm \mathrm{SD} \mathrm{mL} / \mathrm{kg}$ & 27 & $6.8 \pm 2.3$ & 27 & $6.3 \pm 3.1$ & .24 \\
\hline PEEP, median (IQR) $\mathrm{cm} \mathrm{H}_{2} \mathrm{O}$ & 27 & $5(5-7)$ & 27 & $5(5-7)$ & .41 \\
\hline \multicolumn{6}{|l|}{$\begin{array}{l}\text { Laboratory values for subjects with data available } \\
\text { in pre- and post-NAVA periods }\end{array}$} \\
\hline $\mathrm{pH}$, mean $\pm \mathrm{SD}$ & 9 & $7.35 \pm 0.06$ & 9 & $7.36 \pm 0.04$ & .51 \\
\hline $\mathrm{P}_{\mathrm{aCO}_{2}}$, mean $\pm \mathrm{SD} \mathrm{mm} \mathrm{Hg}$ & 9 & $47.6 \pm 8.6$ & 9 & $45.2 \pm 7.8$ & .20 \\
\hline $\mathrm{P}_{\mathrm{aO}_{2}}$, mean $\pm \mathrm{SD} \mathrm{mm} \mathrm{Hg}$ & 8 & $158 \pm 82$ & 8 & $148 \pm 77$ & .67 \\
\hline $\mathrm{P}_{\mathrm{aO}_{2}} / \mathrm{F}_{\mathrm{IO}_{2}}$, median (IQR) mm Hg & 8 & $271(137-419)$ & 8 & $304(97-432)$ & .67 \\
\hline Lactates, mean $\pm \mathrm{SD}$ mmol/L & 7 & $1.15 \pm 0.54$ & 7 & $1.22 \pm 0.4$ & .61 \\
\hline \multicolumn{6}{|l|}{ Laboratory values including all data } \\
\hline $\mathrm{pH}$, mean $\pm \mathrm{SD}$ & 10 & $7.36 \pm 0.06$ & 24 & $7.39 \pm 0.04$ & NA \\
\hline $\mathrm{P}_{\mathrm{aCO}_{2}}$, mean $\pm \mathrm{SD} \mathrm{mm} \mathrm{Hg}$ & 10 & $48.0 \pm 8.2$ & 24 & $45.1 \pm 6.2$ & NA \\
\hline $\mathrm{P}_{\mathrm{aO}_{2}}$, mean $\pm \mathrm{SD} \mathrm{mm} \mathrm{Hg}$ & 8 & $158 \pm 83$ & 18 & $123 \pm 63$ & NA \\
\hline $\mathrm{P}_{\mathrm{aO}_{2}} / \mathrm{F}_{\mathrm{IO}_{2}}$ ratio, median (IQR) $\mathrm{mm} \mathrm{Hg}$ & 8 & $272(137-419)$ & 18 & $206(146-365)$ & NA \\
\hline Lactates, mean $\pm \mathrm{SD}$ mmol/L & 10 & $1.15 \pm 0.46$ & 21 & $1.26 \pm 0.45$ & NA \\
\hline $\begin{array}{l}\text { NAVA = neurally adjusted ventilatory assist } \\
\text { IQR }=\text { interquartile range } \\
\text { NA }=\text { not applicable }\end{array}$ & & & & & \\
\hline
\end{tabular}

jects with blood gas values available both before and during NAVA). During NAVA, data were available in 24 subjects (89\%), exhibiting an average $\mathrm{pH}$ of $7.39 \pm 0.04, \mathrm{a}_{\mathrm{aCO}_{2}}$ of $45.1 \pm 6.2 \mathrm{~mm} \mathrm{Hg}$, and a $\mathrm{P}_{\mathrm{aO}_{2}}$ of $123 \pm 63 \mathrm{~mm} \mathrm{Hg}$.

\section{Discussion}

This study illustrates the clinical feasibility of using NAVA in pediatric subjects after cardiac surgery. This ventilation mode was mostly well tolerated, although used 
in a small proportion of postoperative subjects, which likely represents those undergoing difficult ventilation weaning. When compared to the preceding conventional ventilation, invasive NAVA delivered a similarly effective ventilation with lower positive pressures. Noninvasive NAVA could also be used during ventilation weaning over a large proportion of the total noninvasive ventilation time.

There are few other studies describing the use of NAVA in this particular population. In a recent randomized crossover study, short-term noninvasive NAVA (30 min) after extubation was shown to be effective in 10 infants who underwent cardiac surgery, with decreased work of breathing compared to nasal CPAP. ${ }^{15}$ Berger et al ${ }^{18}$ showed that NAVA had a smaller impact on right ventricular performance when compared with pressure support ventilation in 10 adults during the immediate postoperative cardiac course. Liet et al ${ }^{16}$ compared hemodynamic parameters in a physiologic pilot study of 6 children, finding lower inspiratory pressures and an increase in blood pressure when using NAVA compared to conventional ventilation. Although uncontrolled, our observational study reflects the use of NAVA in actual clinical practice in this specific population, and over longer periods of time. The study population characteristics suggest that invasive NAVA was not routinely used early in the postoperative course, but rather was used in a subset of subjects with protracted weaning and longer stay. One third of the courses, however, occurred in the first $3 \mathrm{~d}$, with a similar impact on ventilation pressure. A more widespread use may have been limited by subjects receiving significant sedation or being extubated early in their postoperative course, as well as the limited availability of NAVA-compatible ventilators during the study period. Importantly, NAVA appeared feasible and well tolerated, with only one subject being ventilated with NAVA for $<2 \mathrm{~h}$. The retrospective design of the study precluded exploring the reasons why NAVA was interrupted.

As we have observed, many previous studies have reported lower inspiratory pressures in children and infants ventilated with NAVA. ${ }^{9,10,19-23}$ The stability of vital signs and blood gas values in our study suggests that this decrease in ventilation pressures was not obtained at the cost of inadequate ventilation. The frequent decrease in positive $\mathrm{P}_{\mathrm{aw}}$ during NAVA while ventilation remains relatively stable is likely secondary to a greater contribution of the patient's own ventilation because of improved patientventilator interactions. This spontaneous ventilation induces patient-generated negative pleural pressure, thereby limiting the need for positive $\mathrm{P}_{\mathrm{aw}}$. This mechanism has been confirmed by Doorduin et $\mathrm{al}^{24}$ who showed that using NAVA in adults with ARDS resulted in lower $\mathrm{P}_{\mathrm{aw}}$ but relatively similar lung-distending pressure. Maintaining the patient's ventilatory efforts and thus negative pleural pressures is an important goal of postoperative care in cardiac subjects, unless significant left ventricle unloading is needed. Rapid extubation is frequently the objective, ${ }^{25}$ but is not always possible. In such cases, NAVA could be physiologically closer to spontaneous ventilation than other modes of assisted ventilation, as suggested by Berger et al, ${ }^{18}$ who observed improved right ventricular performance with NAVA as compared to pressure support ventilation in adults. Our study design did not allow for investigation of heart-lung interactions, and further studies are needed to confirm the positive impact of decreased positive $\mathrm{P}_{\mathrm{aw}}$ in pediatric cardiac patients.

In our study, NAVA was frequently used after several days of ventilation, and we hypothesize that it was specifically considered in subjects presenting protracted weaning. Many studies have shown that NAVA reduces patient-ventilator asynchrony, which is particularly frequent in pediatrics and is associated with adverse outcomes. Moreover, the continuous information provided by the $\mathrm{EA}_{\mathrm{di}}$ signal enables more accurate adjustment of support to avoid over- or under-assist. ${ }^{17}$ This also allows care providers to titrate the level of support to the patient's own work of breathing and progressively lead to spontaneous ventilation. Although unilateral diaphragmatic paralysis can occur after phrenic nerve damage during cardiac surgery, it does not preclude recording an $\mathrm{EA}_{\mathrm{di}}$ signal, and in a context where synchrony is important, NAVA could also be beneficial. In this perspective, we believe that NAVA has the potential to be a useful strategy for the weaning of patients with prolonged ventilation. Evidence of this benefit is still lacking, however. In a randomized, controlled trial involving 170 children, Kallio et al ${ }^{19}$ found that the length of PICU stay of subjects ventilated with NAVA was $23 \mathrm{~h}$ shorter $(P=.10$ in intention-to-treat analysis, $P=.03$ in per-protocol analysis).

Some limitations of our study should be addressed. Our analysis is retrospective and lacks a control group. Although the temporal pattern of ventilatory pressures strongly suggests an impact of the mode of ventilation (Fig. 2), an effect of time on patient evolution cannot be excluded. A significant proportion of our patient population was at risk for deleterious heart-lung interactions, but we were not able to show any improvement in hemodynamic status with decreasing $\mathrm{P}_{\mathrm{aw}}$ for several reasons. First, cardiac output is not routinely monitored in the PICU. Second, at the time of NAVA initiation, the frequency of blood gas analyses was relatively low, and few subjects had available data for pre- and post-NAVA comparisons. The data available in the medical charts did not allow assessment of patient-ventilator synchrony or work of breathing. Moreover, the study sample size did not allow for subgroup analysis according to the type of cardiac surgery. Querying the electronic medical chart nonetheless allowed for all subjects ventilated with NAVA during the study period to be included with only a few missing data. 


\section{NAVA After Pediatric Cardiac Surgery}

Finally, we describe the clinical experience of a single center that has long used NAVA, and the external validity of our results in different contexts is uncertain.

\section{Conclusions}

NAVA represents a ventilation strategy that is feasible in pediatric cardiac intensive care, especially during weaning. In addition to other proven benefits of synchronizing breath delivery with diaphragmatic activity, this strategy is associated with lower airway pressures. Prospective interventional trials are needed to further assess the impact of NAVA in these children, first on short-term cardiac function and pulmonary vascular resistance, and ultimately on important clinical outcomes (eg, comfort, duration of ventilation, and length of stay).

\section{ACKNOWLEDGMENTS}

The authors thank the respiratory therapists, nurses, and fellows of the PICU for their collaboration.

\section{REFERENCES}

1. Pontoppidan H, Geffin B, Lowenstein E. Acute respiratory failure in the adult. N Engl J Med 1972;287(14):690-698.

2. Whittenberger JL, Mc GM, Berglund E, Borst HG. Influence of state of inflation of the lung on pulmonary vascular resistance. J Appl Physiol 1960;15:878-882.

3. West JB, Dollery CT, Naimark A. Distribution of blood flow in isolated lung: relation to vascular and alveolar pressures. J Appl Physiol 1964;19:713-724.

4. Levine S, Nguyen T, Taylor N, Friscia ME, Budak MT, Rothenberg $\mathrm{P}$, et al. Rapid disuse atrophy of diaphragm fibers in mechanically ventilated humans. N Engl J Med 2008;358(13):1327-1335.

5. Putensen C, Zech S, Wrigge H, Zinserling J, Stuber F, Von Spiegel $\mathrm{T}$, et al. Long-term effects of spontaneous breathing during ventilatory support in patients with acute lung injury. Am J Respir Crit Care Med 2001;164(1):43-49.

6. Brochard L, Harf A, Lorino H, Lemaire F. Inspiratory pressure support prevents diaphragmatic fatigue during weaning from mechanical ventilation. Am Rev Respir Dis 1989;139(2):513-521.

7. Sinderby C, Navalesi P, Beck J, Skrobik Y, Comtois N, Friberg S, et al. Neural control of mechanical ventilation in respiratory failure. Nat Med 1999;5(12):1433-1436.

8. Bordessoule A, Emeriaud G, Morneau S, Jouvet P, Beck J. Neurally adjusted ventilatory assist improves patient-ventilator interaction in infants as compared with conventional ventilation. Pediatr Res 2012; 72(2):194-202.

9. Breatnach C, Conlon NP, Stack M, Healy M, O'Hare BP. A prospective crossover comparison of neurally adjusted ventilatory assist and pressure-support ventilation in a pediatric and neonatal intensive care unit population. Pediatr Crit Care Med 2010;11(1):7-11.
10. Bengtsson JA, Edberg KE. Neurally adjusted ventilatory assist in children: an observational study. Pediatr Crit Care Med 2010;11(2): 253-257.

11. Beck J, Reilly M, Grasselli G, Mirabella L, Slutsky AS, Dunn MS, et al. Patient-ventilator interaction during neurally adjusted ventilatory assist in low birth weight infants. Pediatr Res 2009; 65(6):663-668.

12. Clement KC, Thurman TL, Holt SJ, Heulitt MJ. Neurally triggered breaths reduce trigger delay and improve ventilator response times in ventilated infants with bronchiolitis. Intensive Care Med 2011;37(11): 1826-1832.

13. Ducharme-Crevier L, Beck J, Essouri S, Jouvet P, Emeriaud G. Neurally adjusted ventilatory assist (NAVA) allows patient-ventilator synchrony during pediatric noninvasive ventilation: a crossover physiological study. Crit Care 2015;19(1):44.

14. Baudin F, Pouyau R, Cour-Andlauer F, Berthiller J, Robert D, Javouhey E. Neurally adjusted ventilator assist (NAVA) reduces asynchrony during non-invasive ventilation for severe bronchiolitis. Pediatr Pulmonol 2015;50(12):1320-1327.

15. Houtekie L, Moerman D, Bourleau A, Reychler G, Detaille T, Derycke E, et al. Feasibility study on neurally adjusted ventilatory assist in noninvasive ventilation after cardiac surgery in infants. Respir Care 2015;60(7):1007-1014.

16. Liet JM, Barriere F, Gaillard-Le Roux B, Bourgoin P, Legrand A, Joram N. Physiological effects of invasive ventilation with neurally adjusted ventilatory assist (NAVA) in a crossover study. BMC Pediatr 2016;16(1):180.

17. Emeriaud G, Larouche A, Ducharme-Crevier L, Massicotte E, Flechelles O, Pellerin-Leblanc AA, et al. Evolution of inspiratory diaphragm activity in children over the course of the PICU stay. Intensive Care Med 2014;40(11):1718-1726.

18. Berger D, Bloechlinger S, Takala J, Sinderby C, Brander L. Heartlung interactions during neurally adjusted ventilatory assist. Crit Care 2014;18(5):499.

19. Kallio M, Peltoniemi O, Anttila E, Pokka T, Kontiokari T. Neurally adjusted ventilatory assist (NAVA) in pediatric intensive care: a randomized controlled trial. Pediatr Pulmonol 2015;50(1):55-62.

20. Alander M, Peltoniemi O, Pokka T, Kontiokari T. Comparison of pressure-, flow-, and NAVA-triggering in pediatric and neonatal ventilatory care. Pediatr Pulmonol 2012;47(1):76-83.

21. Gentili A, Masciopinto F, Mondardini MC, Ansaloni S, Reggiani ML, Baroncini S. Neurally adjusted ventilatory assist in weaning of neonates affected by congenital diaphragmatic hernia. J Matern Fetal Neonatal Med 2013;26(6):598-602.

22. Stein H, Howard D. Neurally adjusted ventilatory assist in neonates weighing $<1500$ grams: a retrospective analysis. J Pediatr 2012; 160(5):786-789.

23. Lee J, Kim HS, Sohn JA, Lee JA, Choi CW, Kim EK, et al. Randomized crossover study of neurally adjusted ventilatory assist in preterm infants. J Pediatr 2012;161(5):808-813.

24. Doorduin J, Sinderby CA, Beck J, van der Hoeven JG, Heunks LM. Assisted ventilation in patients with acute respiratory distress syndrome: lung-distending pressure and patient-ventilator interaction. Anesthesiology 2015;123(1):181-190.

25. Harris KC, Holowachuk S, Pitfield S, Sanatani S, Froese N, Potts JE, et al. Should early extubation be the goal for children after congenital cardiac surgery? J Thorac Cardiovasc Surg 2014;148(6):2642-2647. 